\title{
Effect of Ankle Stabilization Training Using Biofeedback on Balance Ability and Lower Limb Muscle Activity in Football Players with Functional Ankle Instability
}

\author{
Je-Ho Kim, Yo-Han Uhm \\ Department of Physical Therapy, Sehan University, Yeongam-gun, Jeollanam-do, Korea
}

Purpose: This study focuses on influence of ankle stabilization training on balance ability and lower limb muscle activation of soccer player with functional ankle instability.

Methods: Subjects were grouped into ankle stabilization training group using biofeedback comprised of 15 subjects and general exercise group of 15. The training was conducted for 30 minutes, 3 times a week for 8 weeks in total. All 30 football players conducted plyometric training for 30 minutes before main training. To evaluate balance ability, biorescure was used to measure whole path length and surface area and surface electromyography (EMG) system was used to measure tibialis anterior, tibialis posterior, and soleus to evaluate lower limb muscle activation.

Results: The experiment group showed significant difference to the comparison group in regard of whole path length and surface area which represents balancing capability and muscle activation of tibialis anterior, tibialis posterior, and soleus.

Conclusion: Therefore, ankle stabilization training using biofeedback is more effective in enhancing balance ability and lower limb muscle activation than general exercise.

Keywords: Football, Ankle, Stability, Biofeedback

\section{INTRODUCTION}

The most vulnerable region in sports injuries is the ankle. Once injury occurs, the recurrence rate for ankle sprains is estimated at $80 \%{ }^{1}$ Football players usually suffer from sprains in their ankles through activities such as jumping, landing on one foot, changing directions suddenly or running into other players. ${ }^{2}$ It is reported that the incidence of ankle injury during football games is $12-23 \%$ and $22 \%$ of football players complain of ankle pain although they have no clear memory of the injury. When the injured region of football players was examined, the most common area of damage within the lower limb was the ankle joint. ${ }^{3}$ There are two types of ankle sprains chronic ankle sprains occur due to incomplete rehabilitation after sprain injury, overuse and over training. Acute ankle sprain results in lateral ankle ligament damage caused by sudden

Received May 20, 2016 Revised Jun 15, 2016

Accepted Jun 29, 2016

Corresponding author Yo-Han Uhm

E-mailuhmyo112@naver.com inversion during ankle dorsiflexion. ${ }^{4}$

Chronic ankle instability results in unsteadiness during walking. It is caused when the ankle ligament is chronically ruptured, stretched more than the original length or there is damage of preexisting kinesthetic sense. Forty percent of acute ankle sprains develop into chronic ankle instability. ${ }^{5}$ Chronic ankle instability has two causes, mechanical due to pathologic slackness after ankle ligament injury and functional, which leads to repetitive ankle instability and loss of joint proprioception caused by damage to the nerve root after ligament injury. ${ }^{6}$ Mechanical instability is caused from structural instability which leads to instability of the joint. Functional instability describes the subjective sense of unsteadiness due to recurrent ankle sprain. Damaged proprioception caused by ankle sprains leads to poor sensory feedback for the maintenance of original functioning of the motor center programs which results in

Copylight (C2016 The Korea Society of Physical Therapy

This is an Open Access article distribute under the terms of the Creative Commons Attribution Non-commercial License (Http:// creativecommons.org/license/by-nc/4.o.) which permits unrestricted non-commercial use, distribution, and reproduction in any medium, provided the original work is properly cited. 
functional ankle instability. ${ }^{3}$

Balance ability is an essential element for better performance in sports such as soccer, basketball and gymnastics. In soccer, balancing is particularly important, one leg standing postures are common when performing movements such as shooting and passing and stability of the supporting leg is therefore critical. ${ }^{7}$ Balance ability is predictive of sports injuries, ${ }^{8}$ and it is reported that the lower the balance ability of soccer players, the higher the ankle damage. ${ }^{3}$ Patients with functional ankle instability are unable to balance effectively, this causes abnormal positional reaction patterns, delayed reactions and disorders of stability combined with damage of proprioceptive sense and nerve root control. ${ }^{9}$

There are six essential physical factors involved with sports and muscular strength. These include muscle strength, rapidness, flexibility, endurance, quickness and balance. ${ }^{10}$ Muscular strength is vital to football players because kicking, running, jumping and tackling movements all need strong muscular strength. Therefore, football players require strong muscular strength. ${ }^{11}$ Lower limb muscular strength is one of the most important factors in football players and the powerful lower limbs makes printing and shooting capable. The quadriceps, hamstrings, calf muscles and ankle muscles are predominantly used in soccer. These muscles are closely related with improved athletic performance by maintaining control and physical balance. $^{12}$

Treatment interventions using biofeedback means it is relatively easy to control the experimental environment, selection of location, offers wide training levels to be able to apply subject's ability, rapid and accurate feedback during performance, provides opportunity for self-training in a safe environment and offers an economic training environment. ${ }^{13}$ It has the advantage of improving treatment effects by changing sensory input by training in various environments. ${ }^{14}$ The feedback provided boosts subject's motivation and encourage sactive participation to achieve assignments. ${ }^{15}$ Biofeedback also provides different assignment programs which can focus on the purpose of training to improve physical function. Biofeedback is effective for facilitating motor learning by solving problems voluntarily by receiving vision, sound and somesthesia feedback of error occurring during the performance. ${ }^{13}$ It helps in performing repetitive training and functional exercises in order to improve visual and auditory factors as well as increasing confidence. ${ }^{16}$

Therefore, the purpose of this research is to find out how ankle stabilizing training using biofeedback influences football players with functional ankle instability by measuring muscular activity and balance ability.

\section{METHODS}

\section{Subjects}

We chose thirty football players from the university in Mokpo who agreed with spontaneous participation, had no medical problems with visual and auditory sensation, didn't exceed the normal physiologic range of ankle joint movement, had no other leg injuries except the ankle, had no history of ankle surgery and satisfied more than five items in the Cumberland Ankle Instability Tool. The participants undertook thirty minutes of plyometric training and then the experimental group went through ankle stability training using biofeedback for thirty minutes. The control group then completed an additional thirty minutes of treadmill exercises. The experiment lasted for eight weeks, performed for three days per week for sixty minutes. The experimental group of fifteen people and the control group of fifteen people were randomly selected and the general characteristics of the research subjects are shown in Table 1.

\section{Method}

Modified plyometric training from the Myer and others ${ }^{17}$ method was used for their study and was performed for thirty minutes. We increased the difficulty gradually from simple tasks (running zigzag, running back, vertical jump, agility test, running with side and sprint) simulating a real match situation. Additional ankle stability training using biorescue was executed for the experimental group (Figure 1). Biorescue is composed of a force plate, computer and monitor which provides measurement and training method as op-

Table 1. General characteristics of the subjects

\begin{tabular}{|c|c|c|c|}
\hline & Experiment group $(n=15)$ & Control group $(n=15)$ & \multirow{2}{*}{$p$} \\
\hline & $M \pm S D$ & $M \pm S D$ & \\
\hline Age (year) & $21.36 \pm 2.92$ & $22.04 \pm 4.05$ & 0.46 \\
\hline Height (cm) & $176.91 \pm 5.72$ & $177.24 \pm 6.24$ & 0.87 \\
\hline Weight (kg) & $78.82 \pm 3.93$ & $78.26 \pm 4.42$ & 0.23 \\
\hline CAIT (Score) & $20.54 \pm 3.94$ & $21.56 \pm 4.09$ & 0.68 \\
\hline
\end{tabular}

Mean \pm SD: mean \pm standard deviation.

Experiment group: ankle stabilization training using biofeedback.

Control group: treadmill training.

CAIT: Cumberland ankle instability tool. 


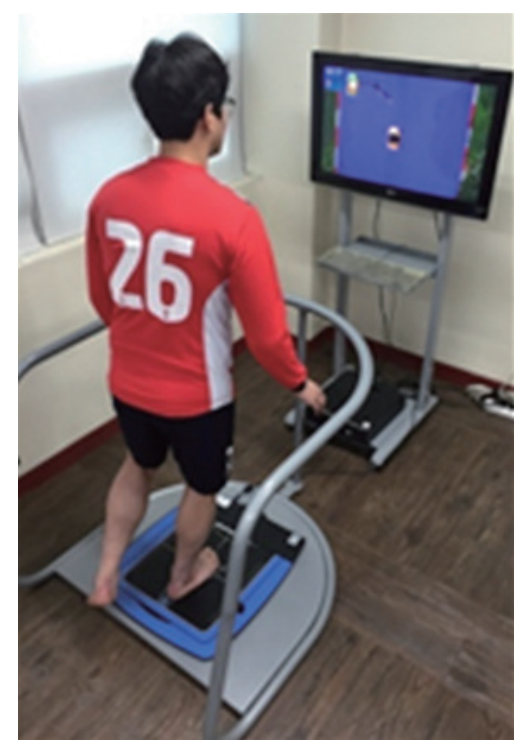

Figure 1. Ankle stabilization training using biofeedback.

tical information, and it offers biofeedback during training (Figure 2). Training is proceeded with six games, and the games were carried out while standing with one leg in the middle of the force plate. The training lasted for thirty minutes with a one-minute break and four minutes training per game. The control group went through thirty minutes of treadmill training (thirty minutes of training was given to the control group) with no additional rest.

\section{Measurement}

\section{1) Balance measuring system}

We used biorescue (RM Ingenierie, France) to analyze the balance ability. This equipment includes a mobile rectangular force plate to stand with two feet and a graduated ruler on the plate indicates the proper position for the feet. To assess balance, we measured the total whole path length and surface area of the center of pressure during standing with standing position for sixty seconds facing forward. For the evaluation, the mean value of the results measured for three times was used.

\section{2) Surface electromyogram system}

In order to measure the muscle activity of the lower limbs, we used an MP100 surface electromyogram system (Biopac system Inc, USA) and we processed the converted digital signal through Acknowledge 3.91 software in individual desktops. We attached bipolar electrodes to the muscles we wanted to measure. We set the sam-
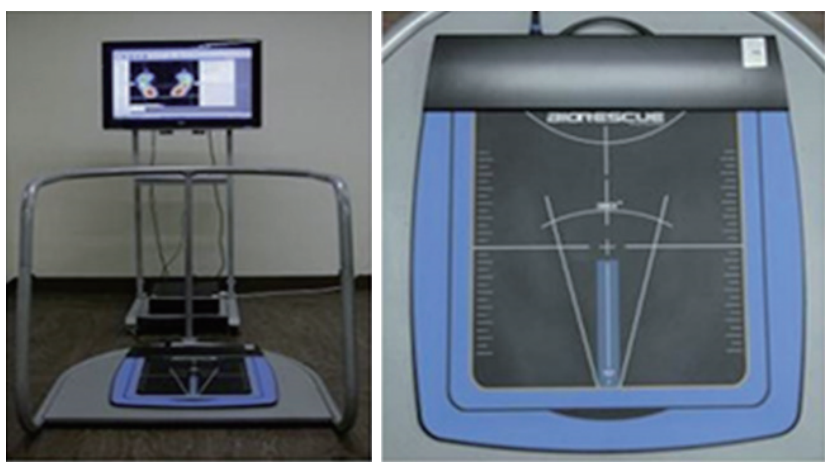

Figure 2. Biorescue

pling rate signal at $1,024 \mathrm{~Hz}$, used notch filter $60 \mathrm{~Hz}$ and band pass filter $30-500 \mathrm{~Hz}$ to minimize noise, and processed the collected signal through root mean square (RMS). To reduce the skin resistance to the surface of electromyogram signal, we removed the hair and rubbed the skin with wipes 3-4 times to get rid of any dead skin cells, and cleaned the skin with alcohol. To standardize the action potential of each muscle, we measured the muscular activity at maximal contracted isometric strength in the Manual Muscle Testing position. After measuring three times for five seconds, we used the average electromyogram signal of the three seconds out of five as \%MVIC except for the first and last second. The measured muscles are anterior tibialis, posterior tibialis and the gastrocnemius. Sekir ${ }^{18}$ emphasized that strengthening these three muscles for patients with functional ankle stability prevents a re-occurrence of the injury and improves athletic performance.

\section{Data analysis}

The resulting analysis was processed through Windows SPSS 18.0 program. Shapiro-wilk was used for the verification of regularity between the experimental group and the control group, and analysis of covariance was used to verify the difference of balance ability and muscular activity between groups following to mediation method. We set the statistical significance level at $\alpha=0.05$.

\section{RESULTS}

\section{Muscle activity}

In the comparison of muscular activity for the anterior tibialis between the two groups before and after the intervention, the experimental group showed $24.66 \pm 3.92 \%-36.12 \pm 4.21 \%$ in muscular ac- 
Table 2. Comparison of muscle activity between groups

\begin{tabular}{|c|c|c|c|c|c|c|}
\hline \multirow{2}{*}{ Muscle } & \multicolumn{2}{|c|}{ Pretest } & \multicolumn{2}{|c|}{ Posttest } & \multirow{2}{*}{$\mathrm{F}$} & \multirow{2}{*}{$\mathrm{p}$} \\
\hline & Experiment group & Control group & Experiment group & Control group & & \\
\hline $\mathrm{TA}(\%)$ & $24.66 \pm 3.92$ & $25.02 \pm 4.37$ & $36.12 \pm 4.21$ & $33.96 \pm 3.19$ & 4.96 & $0.02^{*}$ \\
\hline $\mathrm{TP}(\%)$ & $20.31 \pm 4.25$ & $20.95 \pm 5.04$ & $29.35 \pm 5.13$ & $27.83 \pm 4.25$ & 2.34 & $0.04^{*}$ \\
\hline GCM (\%) & $17.75 \pm 3.93$ & $18.17 \pm 4.06$ & $27.63 \pm 4.64$ & $25.19 \pm 5.25$ & 2.24 & $0.03^{*}$ \\
\hline
\end{tabular}

Mean \pm SD: mean \pm standard deviation, TA: Tibialis anterior, TP: Tibialis posterior, GCM: Gastrocnemius.

${ }^{*} \mathrm{p}<0.05$.

Table 3. Comparison of balance ability between groups

\begin{tabular}{|c|c|c|c|c|c|c|}
\hline \multirow{2}{*}{ Muscle } & \multicolumn{2}{|c|}{ Pretest } & \multicolumn{2}{|c|}{ Posttest } & \multirow{2}{*}{$\mathrm{F}$} & \multirow{2}{*}{$p$} \\
\hline & Experiment group & Control group & Experiment group & Control group & & \\
\hline $\mathrm{SA}\left(\mathrm{mm}^{2}\right)$ & $28.30 \pm 3.03$ & $27.92 \pm 2.97$ & $21.19 \pm 2.34$ & $23.96 \pm 3.01$ & 8.26 & $0.02^{*}$ \\
\hline WPL (mm) & $72.29 \pm 12.34$ & $71.23 \pm 21.33$ & $62.93 \pm 9.92$ & $65.21 \pm 8.97$ & 62.38 & $0.03^{*}$ \\
\hline
\end{tabular}

Mean \pm SD: mean \pm standard deviation, SA: Surface area, WPL: Whole path length. ${ }^{*} p<0.05$.

tivity, $20.31 \pm 4.25 \%-29.35 \pm 5.13 \%$ in posterior tibialis and $17.75 \pm$ $3.93 \%-27.63 \pm 4.64 \%$ in gastrocnemius, which showed a statistically significant difference compared to the control group $(\mathrm{p}<0.05)(\mathrm{Ta}-$ ble 2).

\section{Balance ability}

In the comparison of balance ability between the two groups before and after intervention, the surface area of the experimental group was $28.30 \pm 3.03 \mathrm{~mm}^{2}-21.19 \pm 2.34 \mathrm{~mm}^{2}$ and the whole path length was $72.29 \pm 12.34 \mathrm{~mm}-62.93 \pm 9.90 \mathrm{~mm}$, which also showed a statistically significant difference when compared to the control group $(\mathrm{p}<0.05)$ (Table 3).

\section{DISCUSSION}

This study aims to understand the influence of ankle stability exercise using biofeedback on balance ability and muscle activity in football players with functional ankle instability. The cause of functional ankle instability is weakness of muscular strength and insufficient balance ability. Balance ability plays a basic role in various activities, and is a key factor in successful sport performance. ${ }^{19}$ In addition, insufficiency in proprioception and lack of postural control causes functional ankle instability. ${ }^{6}$ Weakness of muscular activity is related to ankle instability. ${ }^{11}$ When ankle injury occurs the surrounding muscles also show imbalance. ${ }^{20}$ Lower limb strength training has been used in ankle rehabilitation as increased strength in the lower limb can treat and prevent ankle instability. Muscle strength training improves the control of nerve roots and increases muscle mass. ${ }^{21}$ Strengthening of muscles activates the Ia group sense of muscles, activating functional muscle selectively and helps the function of antagonistic muscles. ${ }^{22}$ Changes in the recruitment order of motor units caused by functional ankle instability causes nonfunctional and unnecessary movement in the joint and muscles as it is not able to activate muscles in the proper order. ${ }^{23}$

Although the result of this research showed a statistically significant difference in before and after muscle ability in both the experimental group and the control group, it was more effective in the experimental group. The muscle activity of lower limb results also showed a statistically significant difference before and after the intervention, it was also more effective in the experimental group. $\mathrm{Kim}^{24}$ investigated the balance ability and lower limb muscular strength after performing balance training using a virtual reality program with thirty university students who had functional ankle instability. Balance training using the virtual reality program showed a statistically significant difference in balance ability of ankle dorsiflexion and plantar flexion. The present research also found the same results showing improvements in balance ability and muscle activity in the experimental group with biofeedback.

Training with biofeedback is believed to improve motor performance ability by promoting exercising learning through rapid concentration in a shorter period of time than general training. Chun ${ }^{25}$ studied isokinetic lower limb muscular strength and postural control after performing proprioceptive sensory exercise and muscle strengthening exercises with fifty-eight football players who had 
functional ankle instability. The proprioceptive strength training group showed a significant difference in lower limb strength and postural control capacity. Muscles supporting the ankles are related with balance and postural control. ${ }^{26}$ When the muscles around the ankle are weakened, balance ability is decreased, ${ }^{27}$ and it has been reported that the role of muscles around ankle joints are critical for maintaining balance. ${ }^{28}$ Similar to the previous paper, the present research also shows a statistically significant difference in balance ability and lower limb muscle activity. Unlike the previous study, the present study used biofeedback for the experimental group which revealed that biofeedback training also increased nerve root control ability. It is considered that increases in lower limb muscle activity helps to achieve anticipatory postural control and decreases postural disturbances which produces a positive influence in balance ability. Taube et al. ${ }^{29}$ mentioned that balance ability improves by decreasing unstable movement, restricting spinal reflex excitability such as muscular reflexes during postural control. Kim ${ }^{30}$ reported that among the thirty adults aged twenty years old, the group that used muscle strength training and proprioceptive integration exercises showed a significant difference in isokinetic lower limb muscular strength. This study also showed a statistically significant difference in muscular activity in the lower limb. It is considered that training with biofeedback motivates training and increases muscle activity. It is considered that biofeedback training stimulates proprioception to increase signal transduction of sensory nerves from pressure receptors in the damaged ankle joints with motor control leading to improvement in muscle activity around the ankle joints.

Forestier and Toschi ${ }^{31}$ conducted a study with normal people and patients with chronic ankle instability. The group that used the biomechanical ankle platform system using biofeedback showed a significant difference in muscle activity of the anterior tibialis and peroneus. Furthermore, $\mathrm{Nam}^{32}$ carried out a study with twenty-eight adults with functional ankle instability, the results of which showed a significant difference in muscle activity in the anterior tibialis, soleus and gastrocnemius from the group that used biofeedback training. As shown in the previous study, the results from the present study also show a significant difference in the muscle activity values of the posterior tibialis and gastrocnemius of the experimental group which proves the beneficial treatment effect of ankle stability exercise using biofeedback.
In this research, we investigated the effect of eight weeks of ankle stability exercise using biofeedback on balance ability and lower limb muscle activity in football players with functional ankle instability. Through whole path length and surface area, it is clearly proven that ankle stability exercise using biofeedback is more effective for increasing balance ability than other training. In addition, ankle stabilization exercise using biofeedback is confirmed to be more effective through analyzing the muscle activity value (\%MVIC) of the anterior tibialis, posterior tibialis and the peroneus. Based on our research, we suggest treatment using biofeedback is not only effective in treating the central nervous system but is also an effective treatment for normal people or athletes. We also suggest that more systematic research on the effect of biofeedback for athletes is required.

\section{ACKNOWLEDGMENTS}

The research was has been conducted by the research grant of Sehan university in 2016.

\section{REFERENCES}

1. Hale SA, Hertel J. Reliability and sensitivity of the foot and ankle disability index in subjects with chronic ankle instability. J Athl Train. 2005; 40(1):35-40.

2. Chomiak J, Junge A, Dvorak J. Severe injuries in football players. Influencing factors. Am J Sports Med. 2002;28(5 Suppl):S58-68.

3. Tropp H. Commentary: functional ankle instability revisited. J Athl Train. 2002;37(4):512-15.

4. Hubbard TJ, Kramer LC, Denegar CR et al. Contributing factors to chronic ankle instability. Foot Ankle Int. 2007;28(3):343-54.

5. Valderrabano V, Wiewiorski A, Frigg A et al. Chronic ankle instability. Unfallchirurg. 2007;110(8):691-700.

6. Hertel J. Functional anatomy, pathomechanics, and pathophysiology of lateral ankle instability. J Athl Train. 2002;37(4):364-75.

7. Paillard T, Noe F, Riviere T et al. Postural performance and strategy in the unipedal stance of soccer player at different levels of competition. J Athl Train. 2006;41(2):172-6.

8. McGuine TA, Keene JS. The effects of a balance training program on the risk of ankle sprains in high school athletes. Am J Sports Med. 2006; 34(7):1103-11.

9. Boucher P, Teasdale N, Courtemanche R et al. Postural stability in diabetic polyneuropathy. Diabetes Care. 1995;18(5):638-45.

10. Hoffman JR. Periodized training for the strength/power athlete. NSCA `s Performance Training Journal. 2002;1(9):8-12.

11. Robert K, Karsten K. A soccer-Specific balance training program for hamstring muscle and patellar and achilles tendon injuries. Am J Sports 
Med. 2009;37(7):1384-93.

12. Vanderford M, MEchael CM, William AS et al. Physiological and sportsspecific skill response of olympic youth soccer athletes. J strength cond Res. 2004;18(2):334-42.

13. Jack D, Boian R, Merians AS et al. Virtual reality-enhanced stroke rehabilitation. IEEE Trans Rehabil Eng. 2001;9(3):308-18.

14. Leroux A, Pinet II, Xadeau S. Task-oriented intervention in chronic stroke: Changes in clinical and laboratory measures of balance and mobility. Am J Phys Med Rehabil. 2006;85(10):820-30.

15. Cheng PT, Wu SH, Lian MV et al. Symmetrical body weight distribution on training in stroke patients and its effect on fall prevention. Arch Phys Med Rehabil. 2001;82(12):1650-4.

16. McCombe WS, Liu W, Whitall J. Temporaland spatial control following bilateralversus unilateral training. Hum Mov Sci. 2008;27(5):749-58.

17. Myer GD, Ford KR, McLean SG et al. The effects of plyometric versus dynamic stabilization and balance training on lower extremity biomechanics. The American journal of sports medicine. 2006;34(3):445-55.

18. Sekir G, Hamren J, Milosavljevic S et al. Test-retest reliability of isokinetic training on strength, functionality and proprioception in athletes with functional ankle instability. Knee Surgery Sports and Traumatology Arthroscopy. 2007;15(5):654-64.

19. Erkmen N, Taskin H, Sanioglu A et al. Relationships between balance and functional performance in football players. J Hum Kinet. 2010; 26(1):21-9.

20. Ko YM, Jung MS, Park JW. The relationship between strength balance and joint positon sense related to ankle joint in healthy women. J Kor Phys Ther. 2011;23(2):23-9.

21. Uh BS, Beynnon BD, Helie BV et al. The benefit of a single-leg strength training program for the muscles around the untrained ankle a prospective, randomized, controlled study. 2000;28(4):568-73.

22. Smith BI, Docherty CL, Simon J et al. Ankle strength and force sense after a progressive, 6-week strength-training program in people with func- tional ankle instability. J Athl Train. 2012;47(3):282-8.

23. Song GH, Hwang YT, Park JW et al. The effects of brake pedaling speed on onset time of muscle contraction in the lower extremity during driving task. J Kor Phys Ther. 2011;23(1):7-11.

24. Kim KJ. Effects of virtual reality training program with functional ankle instability. Dongshin University. Dissertation of Master`s Degree. 2015.

25. Chun SY. The effect of proprioceptive exercise program on motor function of lower limb in male soccer players with functional ankle instability. Korea National Sport University. Dissertation of Master`s Degree. 2009.

26. Kim K, Seo SK, Yoon HJ et al. Correlations between muscle strength of the ankle and balance and walking in the elderly. J Kor Phys Ther. 2008;20(1):33-40.

27. Menz HB, Morris ME, Lord SR. Foot and ankle characteristics associated with impaired balance and functional ability in older people. J Gerontol A Biol Sci Med Sci. 2005;60(12):1546-52.

28. Zazulak BT, Hewett TE, Reeves NP et al. Deficits in neuromuscular control of the trunk predict injury risk a prospective biomechanical-epidemiologic study. The American journal of sports medicine. 2007;35(7): 1123-30.

29. Taube W, Gruber M, Gollhofer A. Spinal and supraspinal adaptations associated with balance training and their functional relevance. Acta Physiologica. 2008;193(2):101-16.

30. Kim KJ. The effects of strength and proprioception combined training on functional ankle instability. Dongshin University. Dissertation of Master's D Degree. 2012.

31. Forester N, Toschi P. The effects of an ankle destabilization device on muscular activity while walking. Int J sports Med. 2005;26(6):464-70.

32. Nam SM. The effects on muscle activation and reaction time of the balance trainer controlled knee-joint mobility in functional ankle instability adult males. Daegu University. Dissertation of Master`s Degree. 2015. 